\title{
Effectiveness of Information and Communication Technology on Obesity in Childhood and Adolescence: Systematic Review and Meta-analysis
}

Jihyun Park, MD; Mi-Jeong Park, MD; Young-Gyun Seo, MD, PhD

Department of Family Medicine, Hallym University Sacred Heart Hospital, Anyang, Republic of Korea

\section{Corresponding Author:}

Young-Gyun Seo, MD, PhD

Department of Family Medicine

Hallym University Sacred Heart Hospital

22, Gwanpyeong-ro 170beon-gil

Dongan-gu

Anyang, 14068

Republic of Korea

Phone: 82313803805

Fax: 82313801782

Email: yg035@daum.net

\section{Abstract}

Background: Internet or mobile device use as a form of information and communication technology (ICT) can be more effective in weight loss and weight maintenance than traditional obesity interventions.

Objective: The study aims to assess the effectiveness of child-centered ICT interventions on obesity-related outcomes.

Methods: Articles were retrieved from the Cochrane Central Register of Controlled Trials, Embase, and PubMed web-based databases. We selected randomized controlled trials in which the participants were aged $<18$ years. The primary outcomes were BMI, body weight, BMI $z$-score, waist circumference, and percentage body fat.

Results: In total, 10 of the initial 14,867 studies identified in the databases were selected according to the inclusion criteria. A total of 640 participants were included in the intervention group and 619 in the comparator group. Meta-analyses were conducted considering various subgroups (intervention type, comparator type, target participants, mean age, sex, BMI status, and follow-up period). Overall, ICT interventions demonstrated no significant effect on BMI, body weight, BMI $z$-score, waist circumference, and percentage body fat. Subgroup analyses revealed that the effect of the intervention was statistically significant for the following: $w e b$ intervention (weighted mean difference $[\mathrm{WMD}]=-1.26 \mathrm{~kg} / \mathrm{m}^{2}, 95 \% \mathrm{CI}-2.24$ to -0.28 ), lifestyle modification comparator $(\mathrm{WMD}=-1.75,95 \% \mathrm{CI}-2.76$ to -0.74$)$, intervention involving both boys and girls (WMD $=-1.30,95 \% \mathrm{CI}-2.14$ to -0.46$)$, and intervention involving obesity only (WMD $=-1.92,95 \% \mathrm{CI}-3.75$ to -0.09$)$.

Conclusions: The meta-analysis results for children with obesity who used the web intervention program confirmed significant effects on BMI reduction compared with lifestyle modification. Evidence from the meta-analysis identified internet technology as a useful tool for weight loss in children with obesity.

(J Med Internet Res 2021;23(11):e29003) doi: $10.2196 / 29003$

\section{KEYWORDS}

ICT; eHealth; mHealth; weight loss; obesity; BMI; meta-analysis; randomized controlled trial; children; adolescents; mobile phone

\section{Introduction}

\section{Background}

The prevalence of obesity among children and adolescents worldwide has increased at an alarming rate [1,2]. Children with obesity are now prone to developing diseases that were only observed in adults, including high blood pressure, impaired glucose tolerance, type 2 diabetes mellitus, dyslipidemia, sleep apnea, joint problems, and fatty liver disease [3-5]. In addition, children with obesity may be at risk for a variety of social and 
psychological problems such as low self-esteem, bullying, and discrimination [6].

Given the adverse health outcomes and high prevalence rate, effective interventions are imperative for the management of childhood obesity. Traditionally, many forms of intervention have been attempted to address childhood obesity, and they have mainly comprised lifestyle modifications and mental health care as well as medication and surgical treatment [7-10]. However, some methods have limited indications in children, and their effectiveness is debatable [11,12]. Therefore, it is necessary to develop more efficient and effective approaches for children.

\section{Information and Communication Technology}

Internet and mobile use in childhood and adolescence are already becoming essential elements of young people's lives [13-15], providing several advantages such as learning, information, and entertainment but also causing many problems. Excessive internet and mobile use has resulted in more sedentary behavior, decreased physical activity, and unhealthy dietary patterns, and it is emerging as a social problem that suffices diagnosis as a form of addiction disorder [16]. In addition, studies have reported that increased screen time and obesity are strongly correlated [17].

In contrast, there have been some attempts to use active internet and mobile use during childhood as a means of obesity intervention. Mobile health (mHealth), defined as medical and public health practice supported by mobile devices, such as mobile phones, patient monitoring devices, personal digital assistants, and other wireless devices [18], has the potential to influence a variety of health outcomes and has become a key trend in health service provision during recent years [19]. In addition, previous research has demonstrated that internet-based behavioral interventions have the potential for weight management [20-22].

Information and communication technology (ICT) is a technology that can be used to connect information technologies such as computers and software with communication technologies such as telephones and telecommunication networks. ICT includes cell phone calls, SMS, and apps using a mobile phone, email, and web services using a computer, and telehealth, including health education services, remote monitoring, and remote counseling [23].

\section{Objectives}

Internet or mobile device use as a form of ICT can be more effective in weight loss and weight maintenance than traditional obesity intervention, as it offers benefits in terms of cost, ease of use, accessibility, and time to visit, while improving compliance with prescribed treatments through extensive patient monitoring and continuous support.

Therefore, we conducted a systematic review and meta-analysis to assess the effectiveness of child- and adolescent-centered ICT interventions on obesity-related outcomes.

\section{Methods}

\section{Overview}

We performed a meta-analysis based on the Cochrane Handbook for Systematic Reviews of Interventions [24] and the Centre for Reviews and Dissemination's guidance for undertaking reviews in health care [25]. We reported based on the PRISMA (Preferred Reporting Items for Systematic Reviews and Meta-Analyses) statement [26].

\section{Literature Search}

A systematic search of the effects of ICT on obesity-related outcomes was conducted. We searched the Cochrane Central Register of Controlled Trials, Embase, and PubMed web-based databases and retrieved articles published before January 1, 2021. The search terms used were as follows: ICT OR information and communication technology OR Internet $O R$ web OR social media OR mobile OR smartphone OR application OR app AND obesity OR obese OR weight OR metabolic syndrome. The search was limited to randomized controlled trials (RCTs) and English articles; however, there were no restrictions on the calendar date. Reference lists of the retrieved articles were also reviewed. Information that was unavailable in the selected articles was requested by contacting the relevant authors; however, no response was received.

Two of the authors (JP and MJP) independently reviewed the titles and abstracts after the removal of duplicates. Discrepancies were resolved either by a discussion between the authors or by requesting comments from the third author (YGS). The 3 authors independently analyzed the full text of the remaining articles to determine the final inclusion.

\section{Eligibility Criteria}

We selected the trials to be included in the meta-analysis using the following criteria: (1) the trial was a human RCT written in English, and the full text was available; (2) participants were aged <18 years; (3) the intervention group underwent ICT intervention alone or along with other lifestyle interventions; (4) the comparator group did not undergo ICT intervention; (5) the trial included an assessment of the following primary outcomes: BMI, body weight (BW), BMI z-score, waist circumference (WC), and percentage body fat (\%BF); and (6) mean values of changes from baseline (or postintervention values if not available) with SD (or data suitable for calculating SD: $95 \%$ CI or SE). Uncontrolled, cross-sectional, and animal studies were excluded. The selection criteria did not limit the type of ICT used.

\section{Risk of Bias Assessment}

We used guidelines from the Cochrane Handbook for Systematic Reviews of Interventions to assess the risk of bias in the RCTs [24]. Sources of bias, such as selection bias (random sequence generation and allocation concealment), detection bias (blinding of outcome assessment), attrition bias (incomplete outcome data), and reporting bias (selective reporting) were evaluated. Each domain was assessed in terms of methodological quality, with low or high risk of bias. If data were insufficient to make 
a reasonable judgment, the domain was described as unclear risk of bias.

The risk of bias was reported graphically using Review Manager (RevMan, Version 5.3; Copenhagen: The Nordic Cochrane Center, The Cochrane Collaboration, 2014).

\section{Data Extraction}

Data were independently extracted by 2 authors (JP and YGS) from the selected RCTs. From each RCT, the following data were extracted: name of the first author, year of publication, country where the RCT was performed, sample size, participant-related variables (age, sex, and BMI status), intervention-related variables (ICT type, study duration, target participants, intervention details, comparator details, intervention frequency, and feedback frequency), and treatment effects (mean difference and SD of 2 time point values or mean and SD of postintervention values). The primary outcomes were BMI, $\mathrm{BW}, \mathrm{BMI} z$-score, $\mathrm{WC}$, and \% $\mathrm{BF}$.

\section{Data Synthesis}

The data set was constructed using the mean differences and SDs between the pre- and postintervention values. When the mean difference and SD were not published, the mean and SD of the postintervention values were used. In a meta-analysis, it was possible to combine both the mean differences and the means of postintervention values, assuming that the relative effects assessed by both the mean differences and the means of postintervention values are the same [24]. The final results were calculated and aggregated by one author (YGS).

\section{Meta-analysis}

For the meta-analysis, we used Stata/MP (version 14.0; StataCorp). The weighted mean differences (WMDs) of BMI, $\mathrm{BW}$, BMI $z$-score, WC, and \%BF in the intervention and comparator groups were calculated. We used the Cochran $\mathrm{Q}$ test and $\mathrm{I}^{2}$ test to test the heterogeneity between the study results. For interpretation, $\mathrm{I}^{2}$ values of 25,50 , and 75 were considered to represent low, moderate, and high heterogeneity, respectively [27]. To consider heterogeneity, the DerSimonian and Laird [28] random-effects model for estimating WMD with 95\% CI was used. The effect size and 95\% CI of each study were expressed as forest plots. We checked the symmetry of the funnel plots to evaluate the presence of publication bias. In addition, we used the Egger regression test to evaluate the small study effects [29]. Heterogeneity between studies was analyzed using a meta-regression. We used covariates that may influence the association between ICT and BMI, namely, intervention type (web vs web plus vs app vs app plus), comparator type (control vs print-based vs lifestyle modification), target participants (parents and children vs children vs parents), mean age ( $<10$ vs $\geq 10$ years), sex (boys and girls vs boys vs girls), BMI status (normal to obese vs overweight or obesity vs obesity), and follow-up period ( $\geq 6$ vs $<6$ months). The cutoff for the intervention period (6 months) was based on the transtheoretical model [30]. The statistical significance level was set at $5 \%$. For heterogeneity, a threshold $P$ value of .10 estimated using the Cochran $\mathrm{Q}$ test was considered statistically significant [27].

\section{Results}

\section{Study Selection and Characteristics}

In total, 10 [31-40] of the initial 14,867 studies identified in the databases were selected according to the inclusion criteria, and they contained sufficient data for meta-analysis (Figure 1). The meta-analysis included 13 data sets ( 2 studies each had 2 ICT types [32,34]: web and web plus; one study had two comparator types [38]: lifestyle modification and lifestyle modification plus). A total of 640 participants were included in the intervention group (range of the number of participants, 15-181) and 619 (range of the number of participants, 13-180) in the comparator group. All participants were aged $<18$ years. Of the included studies, one evaluated boys only [40], another evaluated girls only [39], and the other 8 did not differentiate between the sexes of the participants. Six out of 10 studies had intervention periods of 12 weeks, and the remaining 4 had intervention periods $>12$ weeks. Five studies had follow-up periods of 12 weeks, and the remaining 5 had follow-up periods of $>12$ weeks. The frequency of interventions and feedback varied from study to study. The average attendance rate of the participants in the study was $86.49 \%$ (1089/1259). The characteristics of the selected RCTs are summarized in Table 1. 
Figure 1. The PRISMA (Preferred Reporting Items for Systematic Reviews and Meta-Analyses) flow diagram for study selection. ICT: information and communication technology; RCT: randomized controlled trial.

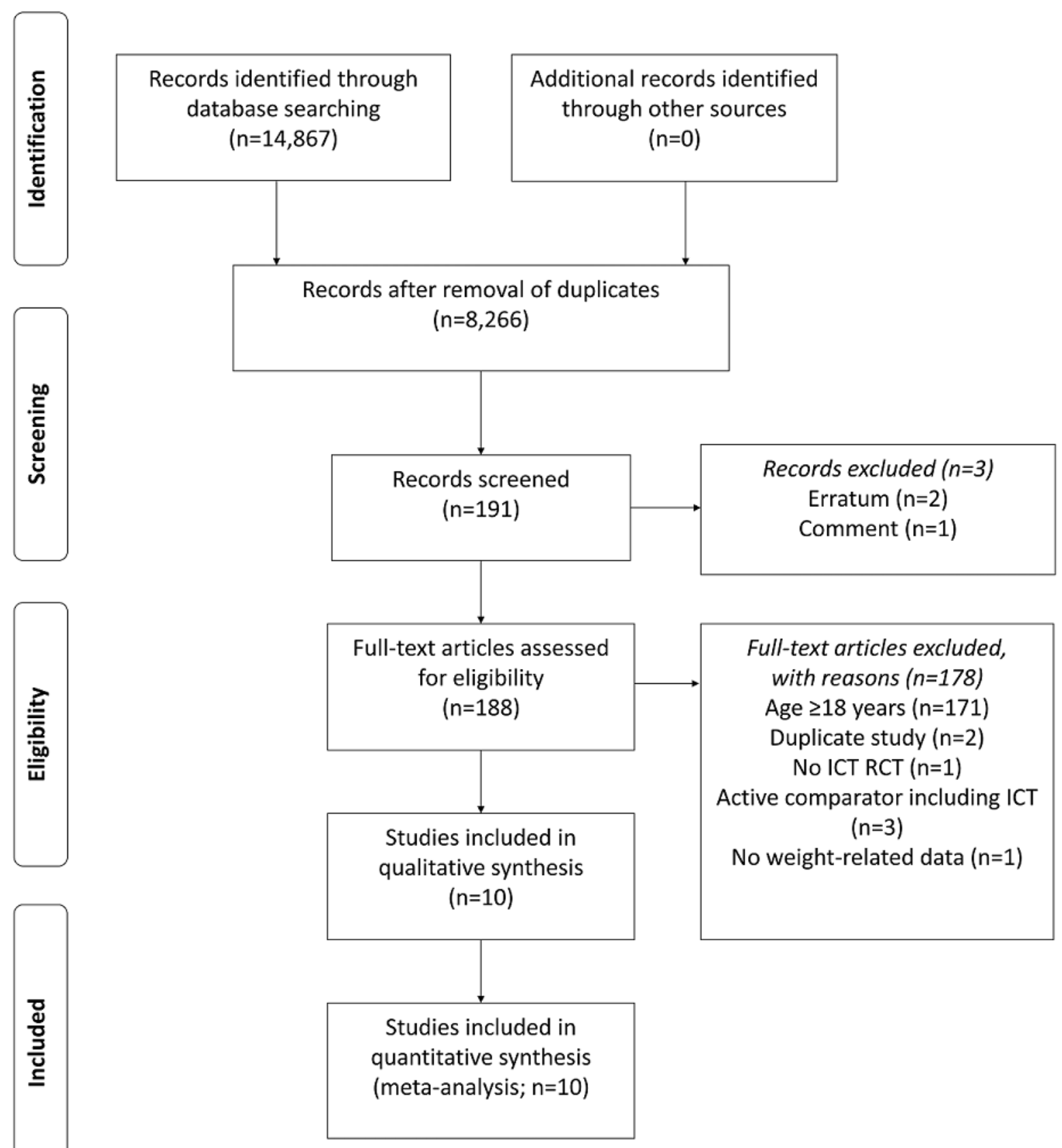


Table 1. Characteristics of the selected randomized clinical trials.

\begin{tabular}{|c|c|c|c|c|c|c|c|c|c|c|c|c|}
\hline $\begin{array}{l}\text { Study } \\
\text { (coun- } \\
\text { try) }\end{array}$ & $\begin{array}{l}\text { Number at } \\
\text { base- } \\
\text { line } \rightarrow \text { fol- } \\
\text { low-up }\end{array}$ & $\begin{array}{l}\text { Age, } \\
\text { range } \\
\text { or } \\
\text { mean } \\
(\mathrm{SD})\end{array}$ & $\begin{array}{l}\text { Sex } \\
(\% \\
\text { males })\end{array}$ & $\begin{array}{l}\text { BMI, } \\
\text { range or } \\
\text { mean } \\
(\mathrm{SD})\end{array}$ & $\begin{array}{l}\text { BMI sta- } \\
\text { tus }\end{array}$ & $\mathrm{ICT}^{\mathrm{a}}$ type & $\begin{array}{l}\text { Interven- } \\
\text { tion dura- } \\
\text { tion } \\
\text { (weeks) }\end{array}$ & $\begin{array}{l}\text { Target } \\
\text { partici- } \\
\text { pants }\end{array}$ & $\begin{array}{l}\text { Interven- } \\
\text { tion details }\end{array}$ & $\begin{array}{l}\text { Comparator } \\
\text { details }\end{array}$ & $\begin{array}{l}\text { Interven- } \\
\text { tion fre- } \\
\text { quency }\end{array}$ & $\begin{array}{l}\text { Feed- } \\
\text { back } \\
\text { fre- } \\
\text { quen- } \\
\text { cy }\end{array}$ \\
\hline $\begin{array}{l}\text { Johans- } \\
\text { son et } \\
\text { al [31] } \\
\text { (Swe- } \\
\text { den) }\end{array}$ & $\begin{array}{l}\mathrm{IG}^{\mathrm{b}} 15 \rightarrow 9 ; \\
\mathrm{CG}^{\mathrm{c}} 13 \rightarrow 9\end{array}$ & $5-12$ & $\begin{array}{l}\text { Male } \\
\text { or fe- } \\
\text { male } \\
(46.4)\end{array}$ & $\begin{array}{l}\text { Boys: } \\
\text { BMI } \\
\geq 98.9 \text { th } \\
\text { per- } \\
\text { centile; } \\
\text { girls: } \\
\text { BMI } \\
\geq 98.6 \text { th } \\
\text { per- } \\
\text { centile }\end{array}$ & Obesity & App & 24 & $\begin{array}{l}\text { Parent } \\
\text { and } \\
\text { chil- } \\
\text { dren }\end{array}$ & $\begin{array}{l}\text { Provement: } \\
\text { display } \\
\text { weight loss } \\
\text { target } \\
\text { curve, ex- } \\
\text { change text } \\
\text { messages; } \\
\text { Lifee Spir- } \\
\text { its+activity } \\
\text { monitor: } \\
\text { increase } \\
\text { motivation } \\
\text { for physi- } \\
\text { cal activity }\end{array}$ & $\begin{array}{l}\mathrm{LSM}^{\mathrm{d}} \text { (improv- } \\
\text { ing dietary } \\
\text { habits and in- } \\
\text { creasing physi- } \\
\text { cal activity to } \\
\text { reduce the de- } \\
\text { gree of obesi- } \\
\text { ty) }\end{array}$ & $\begin{array}{l}\text { Daily } \\
\text { monitor- } \\
\text { ing, mes- } \\
\text { sages } \\
\text { whenever } \\
\text { they felt a } \\
\text { need for } \\
\text { support }\end{array}$ & $\begin{array}{l}\text { At } \\
\text { least } \\
\text { week- } \\
\text { ly }\end{array}$ \\
\hline $\begin{array}{l}\text { Chai } \\
\text { et al } \\
\text { [32] } \\
\text { (Aus- } \\
\text { tralia) }\end{array}$ & $\begin{array}{l}\text { IG (tele- } \\
\text { health) } \\
16 \rightarrow 11 ; \text { IG } \\
\text { (tele- } \\
\text { health+SMS) } \\
15 \rightarrow 10 \text {; CG } \\
15 \rightarrow 15\end{array}$ & $\begin{array}{l}4-11 ; \\
9(2.3)\end{array}$ & $\begin{array}{l}\text { Male } \\
\text { or fe- } \\
\text { male } \\
(59)\end{array}$ & $\begin{array}{l}22.5 \\
(5.1)\end{array}$ & $\begin{array}{l}\text { Over- } \\
\text { weight } \\
\text { or obesi- } \\
\text { ty }\end{array}$ & $\begin{array}{l}\text { Telehealth } \\
\text { or tele- } \\
\text { health+SMS }\end{array}$ & 12 & $\begin{array}{l}\text { Parent } \\
\text { and } \\
\text { chil- } \\
\text { dren }\end{array}$ & $\begin{array}{l}\text { Telehealth } \\
\text { dietitian } \\
\text { consulta- } \\
\text { tion: } \\
\text { semistruc- } \\
\text { tured tele- } \\
\text { health con- } \\
\text { sultations } \\
\text { (approxi- } \\
\text { mately 20 } \\
\text { minutes } \\
\text { each); web- } \\
\text { site } \\
\text { (Back2Ba- } \\
\text { sics Fami- } \\
\text { ly): infor- } \\
\text { mation on } \\
\text { various nu- } \\
\text { trition top- } \\
\text { ic; Face- } \\
\text { book } \\
\text { group: ex- } \\
\text { change } \\
\text { ideas and } \\
\text { informa- } \\
\text { tion related } \\
\text { to the } \\
\text { Back2Ba- } \\
\text { sics Family } \\
\text { website; } \\
\text { SMS to } \\
\text { parents: } \\
\text { targeting } \\
\text { healthy eat- } \\
\text { ing for chil- } \\
\text { dren }\end{array}$ & $\begin{array}{l}\text { Waitlist con- } \\
\text { trol }\end{array}$ & $\begin{array}{l}\text { Tele- } \\
\text { health: } \\
\text { week } 1 \text {, } \\
4 \text {; web- } \\
\text { site: pre- } \\
\text { ferred } \\
\text { time and } \\
\text { frequen- } \\
\text { cy; Face- } \\
\text { book: } \\
\text { weekly; } \\
\text { SMS: 4- } \\
\text { weekly } \\
\text { rotations } \\
\text { of de- } \\
\text { creasing } \\
\text { frequency } \\
\text { (ie, } 5,4 \text {, } \\
3,2 \text { per } \\
\text { week) }\end{array}$ & $\begin{array}{l}\text { Week } \\
1,4\end{array}$ \\
\hline
\end{tabular}




\begin{tabular}{|c|c|c|c|c|c|c|c|c|c|c|c|c|}
\hline $\begin{array}{l}\text { Study } \\
\text { (coun- } \\
\text { try) }\end{array}$ & $\begin{array}{l}\text { Number at } \\
\text { base- } \\
\text { line } \rightarrow \text { fol- } \\
\text { low-up }\end{array}$ & $\begin{array}{l}\text { Age, } \\
\text { range } \\
\text { or } \\
\text { mean } \\
(\mathrm{SD})\end{array}$ & $\begin{array}{l}\text { Sex } \\
(\% \\
\text { males })\end{array}$ & $\begin{array}{l}\text { BMI, } \\
\text { range or } \\
\text { mean } \\
(\mathrm{SD})\end{array}$ & $\begin{array}{l}\text { BMI sta- } \\
\text { tus }\end{array}$ & $\mathrm{ICT}^{\mathrm{a}}$ type & $\begin{array}{l}\text { Interven- } \\
\text { tion dura- } \\
\text { tion } \\
\text { (weeks) }\end{array}$ & $\begin{array}{l}\text { Target } \\
\text { partici- } \\
\text { pants }\end{array}$ & $\begin{array}{l}\text { Interven- } \\
\text { tion details }\end{array}$ & $\begin{array}{l}\text { Comparator } \\
\text { details }\end{array}$ & $\begin{array}{l}\text { Interven- } \\
\text { tion fre- } \\
\text { quency }\end{array}$ & $\begin{array}{l}\text { Feed- } \\
\text { back } \\
\text { fre- } \\
\text { quen- } \\
\text { cy }\end{array}$ \\
\hline $\begin{array}{l}\text { Delisle } \\
\text { Nys- } \\
\text { tröm } \\
\text { et al } \\
\text { [33] } \\
\text { (Swe- } \\
\text { den) }\end{array}$ & $\begin{array}{l}\mathrm{IG} \\
156 \rightarrow 133 ; \\
\mathrm{CG} \\
159 \rightarrow 130\end{array}$ & 4.5 & $\begin{array}{l}\text { Male } \\
\text { or fe- } \\
\text { male } \\
(39)\end{array}$ & $\begin{array}{l}\text { IG: } 15.9 \\
(1.4) ; \\
\text { CG: } \\
15.7 \\
(1.2)\end{array}$ & $\begin{array}{l}\text { Normal } \\
\text { to obesi- } \\
\text { ty }\end{array}$ & App & 24 & Parent & $\begin{array}{l}\text { General in- } \\
\text { formation, } \\
\text { advice, and } \\
\text { evidence- } \\
\text { based } \\
\text { strategies } \\
\text { on how to } \\
\text { change un- } \\
\text { healthy be- } \\
\text { haviors; } \\
\text { register } \\
\text { child's in- } \\
\text { take of } \\
\text { fruits, veg- } \\
\text { etables, } \\
\text { candy, } \\
\text { sweetened } \\
\text { beverages, } \\
\text { and seden- } \\
\text { tary time; } \\
\text { submit } \\
\text { questions } \\
\text { to a dieti- } \\
\text { cian and a } \\
\text { psycholo- } \\
\text { gist to ask } \\
\text { questions } \\
\text { specific to } \\
\text { their child }\end{array}$ & $\begin{array}{l}\text { A pamphlet on } \\
\text { healthy eating } \\
\text { and physical } \\
\text { activity }\end{array}$ & $\begin{array}{l}\text { At least } \\
\text { weekly }\end{array}$ & $\begin{array}{l}\text { Week- } \\
\text { ly }\end{array}$ \\
\hline $\begin{array}{l}\text { Bruñó } \\
\text { et al } \\
\text { [34] } \\
\text { (Spain) }\end{array}$ & $\begin{array}{l}\text { IG (Move It) } \\
18 \rightarrow 15 ; \text { IG } \\
\text { (Move It } \\
\text { plus) } \\
16 \rightarrow 15 ; \text { CG } \\
18 \rightarrow 13\end{array}$ & $\begin{array}{l}9-16 \\
12.6 \\
(1.7)\end{array}$ & $\begin{array}{l}\text { Male } \\
\text { or fe- } \\
\text { male } \\
(57.7)\end{array}$ & $\begin{array}{l}\text { BMI } \\
\geq 85 \text { th } \\
\text { per- } \\
\text { centile }\end{array}$ & $\begin{array}{l}\text { Over- } \\
\text { weight } \\
\text { or obesi- } \\
\text { ty }\end{array}$ & $\begin{array}{l}\text { Web or } \\
\text { web+email }\end{array}$ & 12 & $\begin{array}{l}\text { Chil- } \\
\text { dren }\end{array}$ & $\begin{array}{l}\text { Move It: } \\
\text { web-based } \\
\text { physical } \\
\text { exercise } \\
\text { program } \\
\text { combines } \\
\text { one aerobic } \\
\text { exercise } \\
\text { (brisk } \\
\text { walking) } \\
\text { and 10 } \\
\text { muscular } \\
\text { strength ex- } \\
\text { ercises; } \\
\text { Move It } \\
\text { plus: move } \\
\text { It+weekly } \\
\text { reminder } \\
\text { and motiva- } \\
\text { tional } \\
\text { emails }\end{array}$ & $\begin{array}{l}\text { The same exer- } \\
\text { cise program } \\
\text { as the interven- } \\
\text { tion group by } \\
\text { a written } \\
\text { guide }\end{array}$ & $\begin{array}{l}60 \text { ses- } \\
\text { sions dis- } \\
\text { tributed } \\
\text { over } 3 \\
\text { months, } \\
\text { with } 5 \\
\text { weekly } \\
\text { sessions } \\
\text { of } 60 \\
\text { minutes } \\
\text { each }\end{array}$ & $\begin{array}{l}\text { Week- } \\
\text { ly }\end{array}$ \\
\hline
\end{tabular}




\begin{tabular}{|c|c|c|c|c|c|c|c|c|c|c|c|c|}
\hline $\begin{array}{l}\text { Study } \\
\text { (coun- } \\
\text { try) }\end{array}$ & $\begin{array}{l}\text { Number at } \\
\text { base- } \\
\text { line } \rightarrow \text { fol- } \\
\text { low-up }\end{array}$ & $\begin{array}{l}\text { Age, } \\
\text { range } \\
\text { or } \\
\text { mean } \\
(\mathrm{SD})\end{array}$ & $\begin{array}{l}\text { Sex } \\
(\% \\
\text { males })\end{array}$ & $\begin{array}{l}\text { BMI, } \\
\text { range or } \\
\text { mean } \\
(\mathrm{SD})\end{array}$ & $\begin{array}{l}\text { BMI sta- } \\
\text { tus }\end{array}$ & $\mathrm{ICT}^{\mathrm{a}}$ type & $\begin{array}{l}\text { Interven- } \\
\text { tion dura- } \\
\text { tion } \\
\text { (weeks) }\end{array}$ & $\begin{array}{l}\text { Target } \\
\text { partici- } \\
\text { pants }\end{array}$ & $\begin{array}{l}\text { Interven- } \\
\text { tion details }\end{array}$ & $\begin{array}{l}\text { Comparator } \\
\text { details }\end{array}$ & $\begin{array}{l}\text { Interven- } \\
\text { tion fre- } \\
\text { quency }\end{array}$ & $\begin{array}{l}\text { Feed- } \\
\text { back } \\
\text { fre- } \\
\text { quen- } \\
\text { cy }\end{array}$ \\
\hline $\begin{array}{l}\text { Rerk- } \\
\text { suppa- } \\
\text { phol } \\
\text { and } \\
\text { Rerk- } \\
\text { suppa- } \\
\text { phol } \\
\text { [35] } \\
\text { (Thai- } \\
\text { land) }\end{array}$ & $\begin{array}{l}\mathrm{IG} \\
111 \rightarrow 111 ; \\
\mathrm{CG} \\
107 \rightarrow 106\end{array}$ & $\begin{array}{l}10.7 \\
(3.1)\end{array}$ & $\begin{array}{l}\text { Male } \\
\text { or fe- } \\
\text { male } \\
(49)\end{array}$ & $\begin{array}{l}8.36 \\
\mathrm{~kg} / \mathrm{m}^{2} \\
(\mathrm{IQR} \\
16.08- \\
22.09 \\
\left.\mathrm{~kg} / \mathrm{m}^{2}\right)\end{array}$ & $\begin{array}{l}\text { Normal } \\
\text { to obesi- } \\
\text { ty }\end{array}$ & Web & 16 & $\begin{array}{l}\text { Chil- } \\
\text { dren }\end{array}$ & $\begin{array}{l}\text { Personal } \\
\text { data collec- } \\
\text { tion, anthro- } \\
\text { pometric } \\
\text { variables } \\
\text { and the in- } \\
\text { terpretation } \\
\text { of nutrition- } \\
\text { al status, } \\
\text { informa- } \\
\text { tion related } \\
\text { to healthy } \\
\text { nutrition, } \\
\text { food habits } \\
\text { and physi- } \\
\text { cal activity } \\
\text { by web }\end{array}$ & $\begin{array}{l}\text { The same pro- } \\
\text { gram as the in- } \\
\text { tervention } \\
\text { group by } \\
\text { trained re- } \\
\text { search assis- } \\
\text { tants }\end{array}$ & Monthly & $\begin{array}{l}\text { Month- } \\
\text { ly }\end{array}$ \\
\hline $\begin{array}{l}\text { Mameli } \\
\text { et al } \\
{[36]} \\
\text { (Italy) }\end{array}$ & $\begin{array}{l}\text { IG } 23 \rightarrow 16 \\
\text { CG } 20 \rightarrow 14\end{array}$ & $10-17$ & $\begin{array}{l}\text { Male } \\
\text { or fe- } \\
\text { male } \\
(61.9)\end{array}$ & $\begin{array}{l}\text { BMI } \\
\geq 95 \text { th } \\
\text { per- } \\
\text { centile }\end{array}$ & Obesity & $\begin{array}{l}\text { App+wrist } \\
\text { band }\end{array}$ & 12 & $\begin{array}{l}\text { Chil- } \\
\text { dren }\end{array}$ & $\begin{array}{l}\text { App: mea- } \\
\text { sure energy } \\
\text { intake; } \\
\text { wrist band: } \\
\text { measure } \\
\text { energy ex- } \\
\text { penditure; } \\
\text { SMS: feed- } \\
\text { back }\end{array}$ & $\begin{array}{l}\text { LSM (the } \\
\text { Mediterranean } \\
\text { diet and in- } \\
\text { struction to } \\
\text { practice physi- } \\
\text { cal activity } \\
\text { and minimize } \\
\text { sedentary ac- } \\
\text { tivity) }\end{array}$ & $\begin{array}{l}\text { Data ob- } \\
\text { tained by } \\
\text { the wrist } \\
\text { band and } \\
\text { app were } \\
\text { made } \\
\text { available } \\
\text { daily }\end{array}$ & $\begin{array}{l}\text { Week- } \\
\text { ly }\end{array}$ \\
\hline $\begin{array}{l}\text { Mo- } \\
\text { hammed } \\
\text { Nawi } \\
\text { and } \\
\text { Che } \\
\text { Ja- } \\
\text { maludin } \\
\text { [37] } \\
\text { (Makysia) }\end{array}$ & $\begin{array}{l}\text { IG } 47 \rightarrow 47 \\
\text { CG } 50 \rightarrow 50\end{array}$ & 16 & $\begin{array}{l}\text { Male } \\
\text { or fe- } \\
\text { male } \\
(56.7)\end{array}$ & $\begin{array}{l}\text { BMI } \\
>25 \\
\mathrm{~kg} / \mathrm{m}^{2}\end{array}$ & $\begin{array}{l}\text { Over- } \\
\text { weight } \\
\text { or obesi- } \\
\text { ty }\end{array}$ & Web & 12 & $\begin{array}{l}\text { Chil- } \\
\text { dren }\end{array}$ & $\begin{array}{l}\text { Informa- } \\
\text { tion on } \\
\text { healthy } \\
\text { lifestyle, } \\
\text { diet, and } \\
\text { ways to } \\
\text { overcome } \\
\text { obesity, } \\
\text { discussion } \\
\text { by web }\end{array}$ & $\begin{array}{l}\text { The same in- } \\
\text { formation as } \\
\text { the interven- } \\
\text { tion group by } \\
\text { the pamphlets }\end{array}$ & $\begin{array}{l}\text { Weigh } \\
\text { and calcu- } \\
\text { late BMI } \\
\text { every } 2 \\
\text { weeks; } \\
\text { notified } \\
\text { with any } \\
\text { updates } \\
\text { and infor- } \\
\text { mation }\end{array}$ & $\begin{array}{l}\text { Chat } \\
\text { ses- } \\
\text { sions } \\
\text { on the } \\
\text { web- } \\
\text { site; } \\
\text { moni- } \\
\text { toring } \\
\text { nega- } \\
\text { tive } \\
\text { com- } \\
\text { ment } \\
\text { by ad- } \\
\text { min }\end{array}$ \\
\hline $\begin{array}{l}\text { Abra- } \\
\text { ham et } \\
\text { al [38] } \\
\text { (Chi- } \\
\text { na) }\end{array}$ & $\begin{array}{l}\text { IG } 16 \rightarrow 16 ; \\
\text { CG }\left(s^{2} P^{e}\right) \\
16 \rightarrow 16 ; C G \\
\text { (control) } \\
16 \rightarrow 16\end{array}$ & $12-18$ & $\begin{array}{l}\text { Male } \\
\text { or fe- } \\
\text { male } \\
(60.4)\end{array}$ & $\begin{array}{l}\text { BMI } \\
\geq 95 \text { th } \\
\text { per- } \\
\text { centile }\end{array}$ & Obesity & $\begin{array}{l}\text { Web+cell } \\
\text { phone } \\
\text { calls+SMS }\end{array}$ & 12 & $\begin{array}{l}\text { Chil- } \\
\text { dren }\end{array}$ & $\begin{array}{l}\text { Internet- } \\
\text { based cur- } \\
\text { riculum } \\
\text { (nutrition } \\
\text { and physi- } \\
\text { cal activi- } \\
\text { ty); cell } \\
\text { phone fol- } \\
\text { low-up; } \\
\text { weekly } \\
\text { semiperson- } \\
\text { alized SMS }\end{array}$ & $\begin{array}{l}\text { Control: usual } \\
\text { care consisted } \\
\text { of a focused } \\
\text { dietary and } \\
\text { physical activ- } \\
\text { ity history, } \\
\text { medical histo- } \\
\text { ry, physical } \\
\text { examination, } \\
\text { laboratory } \\
\text { screening and } \\
\text { obesity coun- } \\
\text { seling; sLMP: } \\
\text { usual } \\
\text { care+four } \\
\text { meetings with } \\
\text { a nutritionist } \\
\text { over } 3 \text { months }\end{array}$ & $\begin{array}{l}\text { Goal set- } \\
\text { ting: } \\
\text { monthly }\end{array}$ & $\begin{array}{l}\text { Week- } \\
\text { ly } \\
\text { SMS }\end{array}$ \\
\hline
\end{tabular}




\begin{tabular}{|c|c|c|c|c|c|c|c|c|c|c|c|c|}
\hline $\begin{array}{l}\text { Study } \\
\text { (coun- } \\
\text { try) }\end{array}$ & $\begin{array}{l}\text { Number at } \\
\text { base- } \\
\text { line } \rightarrow \text { fol- } \\
\text { low-up }\end{array}$ & $\begin{array}{l}\text { Age, } \\
\text { range } \\
\text { or } \\
\text { mean } \\
(\mathrm{SD})\end{array}$ & $\begin{array}{l}\text { Sex } \\
(\% \\
\text { males })\end{array}$ & $\begin{array}{l}\mathrm{BMI}, \\
\text { range or } \\
\text { mean } \\
(\mathrm{SD})\end{array}$ & $\begin{array}{l}\text { BMI sta- } \\
\text { tus }\end{array}$ & $\mathrm{ICT}^{\mathrm{a}}$ type & $\begin{array}{l}\text { Interven- } \\
\text { tion dura- } \\
\text { tion } \\
\text { (weeks) }\end{array}$ & $\begin{array}{l}\text { Target } \\
\text { partici- } \\
\text { pants }\end{array}$ & $\begin{array}{l}\text { Interven- } \\
\text { tion details }\end{array}$ & $\begin{array}{l}\text { Comparator } \\
\text { details }\end{array}$ & $\begin{array}{l}\text { Interven- } \\
\text { tion fre- } \\
\text { quency }\end{array}$ & $\begin{array}{l}\text { Feed- } \\
\text { back } \\
\text { fre- } \\
\text { quen- } \\
\text { cy }\end{array}$ \\
\hline $\begin{array}{l}\text { Nollen } \\
\text { et al } \\
\text { [39] } \\
\text { (Unit- } \\
\text { ed } \\
\text { States) }\end{array}$ & $\begin{array}{l}\text { IG } 26 \rightarrow 22 ; \\
\text { CG } 25 \rightarrow 22\end{array}$ & $\begin{array}{l}9-14 ; \\
11.3 \\
(1.6)\end{array}$ & $\begin{array}{l}\mathrm{Fe}- \\
\text { male } \\
(0)\end{array}$ & $\begin{array}{l}23.7 \\
(5.7)\end{array}$ & $\begin{array}{l}\text { Normal } \\
\text { to obesi- } \\
\text { ty }\end{array}$ & $\begin{array}{l}\text { Standalone } \\
\text { mobile app }\end{array}$ & 12 & $\begin{array}{l}\text { Chil- } \\
\text { dren }\end{array}$ & $\begin{array}{l}\text { Set } 2 \text { daily } \\
\text { goals and } \\
\text { an accom- } \\
\text { panying } \\
\text { plan for im- } \\
\text { proving the } \\
\text { behavior, } \\
\text { and feed- } \\
\text { back and } \\
\text { reinforce- } \\
\text { ment on } \\
\text { goal-attain- } \\
\text { ment }\end{array}$ & $\begin{array}{l}\text { The same con- } \\
\text { tents as the in- } \\
\text { tervention } \\
\text { group by the } \\
\text { manuals }\end{array}$ & $\begin{array}{l}\text { Self-moni- } \\
\text { tor } \\
\text { progress } \\
\text { toward } \\
\text { their } \\
\text { goals at } 5 \\
\text { times a } \\
\text { day }\end{array}$ & $\begin{array}{l}\text { Five } \\
\text { times } \\
\text { a day }\end{array}$ \\
\hline $\begin{array}{l}\text { Smith } \\
\text { et al } \\
\text { [40] } \\
\text { (Aus- } \\
\text { tralia) }\end{array}$ & $\begin{array}{l}\mathrm{IG} \\
181 \rightarrow 139 ; \\
\mathrm{CG} \\
180 \rightarrow 154\end{array}$ & $\begin{array}{l}12-14 \\
12.7 \\
(0.5)\end{array}$ & $\begin{array}{l}\text { Male } \\
(100)\end{array}$ & $\begin{array}{l}20.5 \\
(4.1)\end{array}$ & $\begin{array}{l}\text { Normal } \\
\text { to obesi- } \\
\text { ty }\end{array}$ & App & 20 & $\begin{array}{l}\text { Chil- } \\
\text { dren }\end{array}$ & $\begin{array}{l}\text { Supple- } \\
\text { ment the } \\
\text { delivery of } \\
\text { enhanced } \\
\text { school } \\
\text { sport and } \\
\text { interactive } \\
\text { sessions by } \\
\text { providing } \\
\text { participants } \\
\text { with physi- } \\
\text { cal activity } \\
\text { monitor- } \\
\text { ing, record- } \\
\text { ing of fit- } \\
\text { ness chal- } \\
\text { lenge re- } \\
\text { sults, tai- } \\
\text { lored moti- } \\
\text { vational } \\
\text { messaging, } \\
\text { peer assess- } \\
\text { ment of re- } \\
\text { sistance } \\
\text { training } \\
\text { skills, and } \\
\text { goal setting } \\
\text { for physi- } \\
\text { cal activity } \\
\text { and screen- } \\
\text { time }\end{array}$ & $\begin{array}{l}\text { Participate in } \\
\text { usual practice } \\
\text { (regularly } \\
\text { scheduled } \\
\text { school sports } \\
\text { and physical } \\
\text { education } \\
\text { lessons) }\end{array}$ & $\begin{array}{l}\text { Tailored } \\
\text { motiva- } \\
\text { tional and } \\
\text { informa- } \\
\text { tional } \\
\text { push } \\
\text { prompt } \\
\text { messages }\end{array}$ & $\begin{array}{l}\text { Peer } \\
\text { assess- } \\
\text { ment }\end{array}$ \\
\hline
\end{tabular}

ICT: information and communication technology.

${ }^{\mathrm{b}} \mathrm{IG}$ : intervention group.

${ }^{\mathrm{c}} \mathrm{CG}$ : comparator group.

${ }^{\mathrm{d}}$ LSM: lifestyle modification.

esLMP: simplified lifestyle modification program.

\section{ICT Interventions}

Of the included 10 studies, 5 (50\%) were web-based interventions, and the other $5(50 \%)$ were app-based interventions.

Two of the 5 web-based intervention studies were web interventions, one for providing web-based nutrition and physical activity information for children [35] and one for providing web-based nutrition information for children [37].

One of the 5 web-based intervention studies was web or web plus intervention, which provided web-based physical exercise programs and motivational emails for children [34]. Two of the 5 web-based intervention studies were web plus interventions, one for providing web-based nutrition and physical activity information as well as SMS feedback for children [38] and one for providing web-based telehealth dietitian consultation, nutrition information, and SMS feedback for parents and children [32]. 
Four of the 5 app-based intervention studies were app interventions, one for providing app-based weight loss target curve, physical activity information for parents and children [31], one for providing app-based nutrition information for parents [33], one for providing app-based nutrition and screen-time information for children [39], and one for providing app-based physical activity and screen-time information for children [40]. One of the 5 app-based intervention studies was app plus intervention, which provided app-based nutrition and physical activity information and SMS feedback for children [36].

\section{Risk of Bias}

Participant blinding was not possible because of the characteristics of the intervention. Therefore, performance bias was not considered in the risk of bias assessment. There was some risk of bias in the individual studies. Two studies lacked sufficient data to evaluate the randomization sequence generation. Four studies lacked sufficient data to evaluate allocation concealment. Three studies indicated blinding of outcome assessment; however, one study stated that assessors were not blinded at follow-up. Two studies lacked sufficient data to evaluate the attrition bias. Three studies lacked sufficient information to evaluate the study protocol, and one study did not report all of the information. The risk of bias assessment is reported graphically in Figure S1 in Multimedia Appendix 1 [31-40].

\section{Synthesis of Results}

To evaluate the overall intervention effect, we calculated the mean difference in BMI for each study. Figure 2 shows the effect size for each study and the overall effect size. The intervention demonstrated no significant effect on BMI (WMD $=-0.52 \mathrm{~kg} / \mathrm{m}^{2}, 95 \% \mathrm{CI}-1.17$ to 0.13 ). Categorization of target participants, mean age, and BMI appeared to have moderate to high heterogeneity. Categorization of the follow-up period appeared to have low to moderate heterogeneity. For all other categories, the heterogeneity was low. We also calculated mean differences in BW, BMI $z$-score, WC, and \%BF to determine the overall intervention effect, and no significant intervention effects were identified for $\mathrm{BW}(\mathrm{WMD}=-0.22 \mathrm{~kg}$, $95 \% \mathrm{CI}-1.05$ to 0.62$)$, BMI $z$-score $(\mathrm{WMD}=-0.22,95 \% \mathrm{CI}$ -0.49 to 0.04$)$, WC (WMD $=-1.70 \mathrm{~cm}, 95 \% \mathrm{CI}-3.91$ to 0.51$)$, and $\% \mathrm{BF}(\mathrm{WMD}=-0.00 \%, 95 \% \mathrm{CI}-0.07$ to 0.07 ; Figure $\mathrm{S} 2$ in Multimedia Appendix 1).

Figure 2. Forest plot for changes in BMI. Meta-analysis of the effect of the information and communication technology (ICT) on BMI $\left(\mathrm{kg} / \mathrm{m}^{2}\right)$. The mean difference for each study reporting changes in BMI is depicted along with the $95 \%$ CI. The random-effects model was used to estimate the weighted mean differences with 95\% CIs. Negative values favor ICT because the ICT intervention group experienced more BMI reduction than the comparator group did. WMD: weighted mean difference.

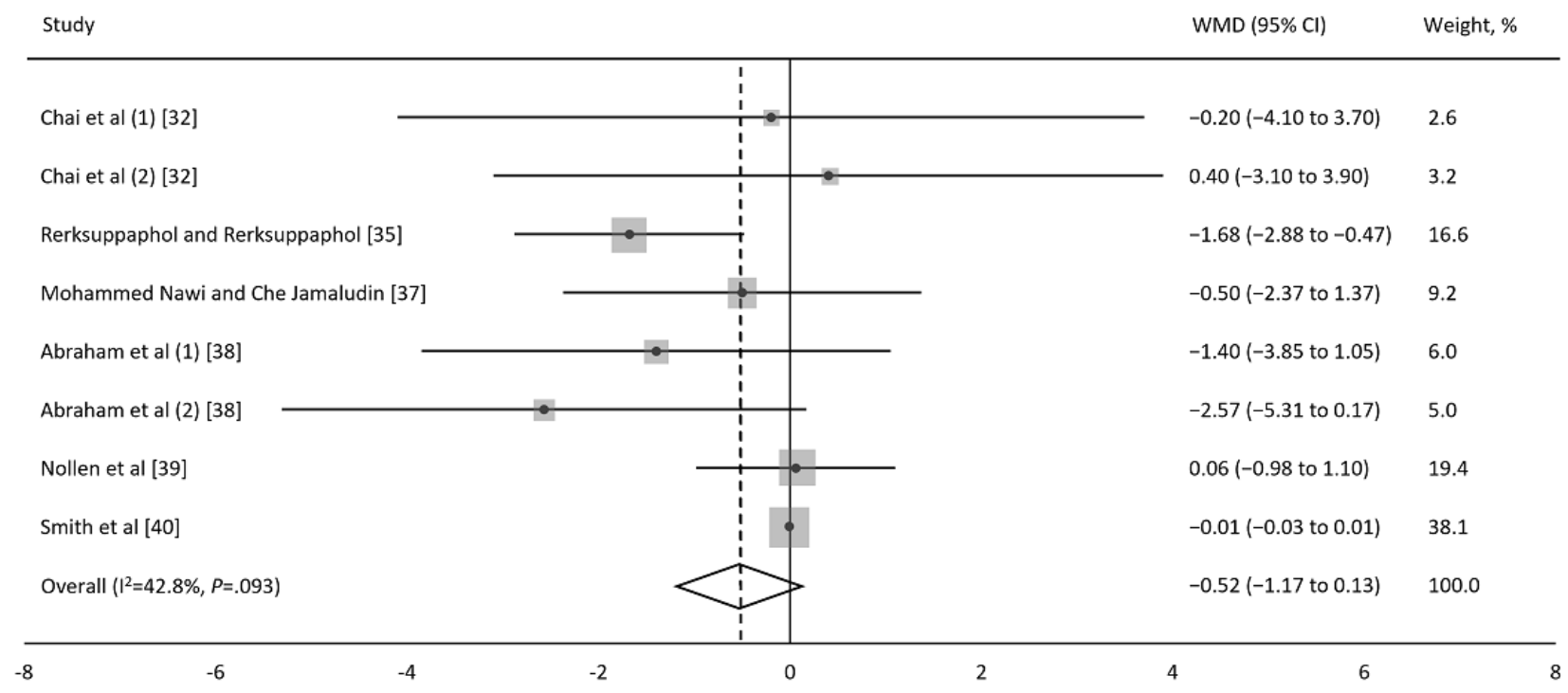

\section{Publication Bias}

To verify possible publication bias, we plotted the effect size no statistically significant publication bias according to the Egger test $(P=.09)$. against the SE to generate a funnel plot (Figure 3). There was 
Figure 3. Funnel plot for changes in BMI. The funnel plots of SE of weighted mean difference (WMD) against WMD for BMI to assess for publication bias. WMD: weighted mean difference.

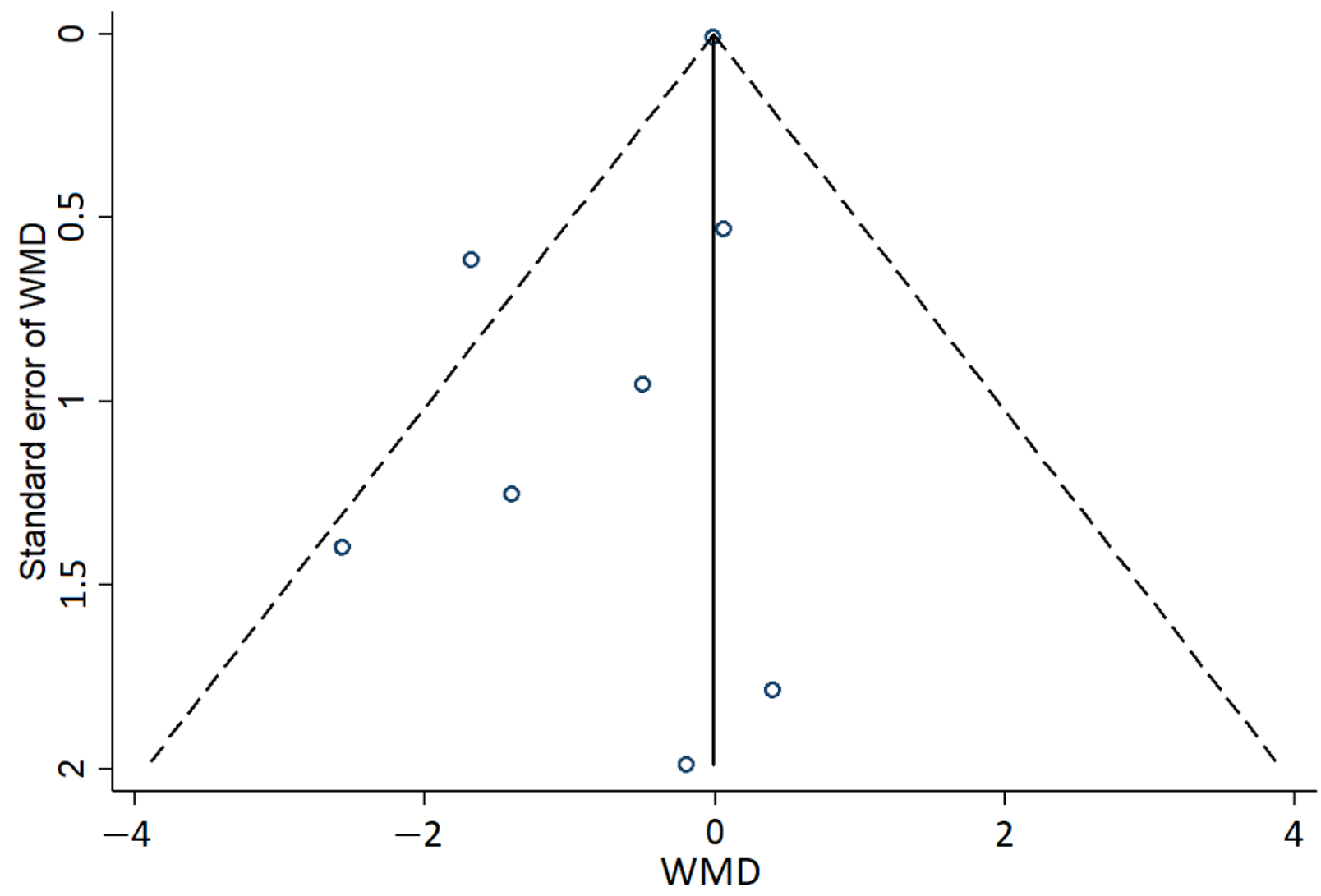

\section{Meta-regression}

The results of the simple meta-regression analysis were significant for categorical covariates of intervention type $(\beta=.69$, $95 \%$ CI 0.10 to 1.28$)$ and comparator type $(\beta=-.73,95 \% \mathrm{CI}$ -1.39 to -0.07$)$. A subgroup analysis by intervention type revealed that the intervention effect was statistically significant only in the $w e b$ intervention (WMD $=-1.26 \mathrm{~kg} / \mathrm{m}^{2}, 95 \% \mathrm{CI}-2.24$ to -0.28). A subgroup analysis by comparator type demonstrated that the intervention effect was statistically significant only in the lifestyle modification comparator (WMD $=-1.75 \mathrm{~kg} / \mathrm{m}^{2}, 95 \%$ CI -2.76 to -0.74$)$. A subgroup analysis by sex revealed that the intervention effect was statistically significant only in the intervention involving both boys and girls (WMD $=-1.30 \mathrm{~kg} / \mathrm{m}^{2}$, $95 \%$ CI -2.14 to -0.46 ). In addition, a subgroup analysis by BMI status demonstrated that the intervention effect was statistically significant only in the intervention involving obesity only (WMD $=-1.92 \mathrm{~kg} / \mathrm{m}^{2}, 95 \% \mathrm{CI}-3.75$ to -0.09 ; Figure S3 in Multimedia Appendix 1).

Subgroup analyses were also performed for other outcome variables, and the results of subgroups containing only one study data were excluded. For BW, subgroup analysis revealed that the intervention effect was statistically significant only in the web intervention (WMD=-1.21 kg, 95\% CI -2.36 to -0.06 ) and the intervention involving a follow-up period $<6$ months $(\mathrm{WMD}=-0.87 \mathrm{~kg}, 95 \% \mathrm{CI}-1.73$ to -0.01 ; Figure $\mathrm{S} 4$ in
Multimedia Appendix 1). For BMI $z$-score, subgroup analysis demonstrated that the intervention effect was statistically significant only in the intervention targeting parents and children $(\mathrm{WMD}=-0.23,95 \% \mathrm{CI}-0.37$ to -0.08 ) and that where the average age of participants was $<10$ years $(\mathrm{WMD}=-0.23$, 95\% CI -0.37 to -0.08; Figure S5 in Multimedia Appendix 1). For WC, subgroup analysis revealed that the intervention effect was statistically significant only in the web plus intervention (WMD $=-4.88 \mathrm{~cm}, 95 \% \mathrm{CI}-8.93$ to -0.83 ), lifestyle modification and control comparator $(\mathrm{WMD}=-3.58 \mathrm{~cm}, 95 \%$ $\mathrm{CI}-6.30$ to -0.85 and $\mathrm{WMD}=0.50 \mathrm{~cm}, 95 \%$ CI 0.43 to 0.57 , respectively), intervention involving both boys and girls (WMD $=-2.95 \mathrm{~cm}, 95 \% \mathrm{CI}-5.18$ to -0.71 ), and intervention involving obesity only (WMD $=-5.50 \mathrm{~cm}, 95 \% \mathrm{CI}-9.89$ to -1.12; Figure S6 in Multimedia Appendix 1). Subgroup analyses demonstrated no statistically significant effect on \%BF (Figure S7 in Multimedia Appendix 1).

\section{Behavioral Change}

Nine of the included 10 studies reported the initial results, and only one study reported the results of an additional 6 months follow-up after reporting the initial results of a 6-month intervention [33].

Five of the included 10 studies also reported results on behavioral changes. Some studies have shown that there were no significant intervention effects on sedentary time [33], physical activity $[33,38]$, consumption of fruits and vegetables, 
and sugar-sweetened beverages [33,39]. However, one study reported that screen-time and sugar-sweetened beverage consumption [40] were improved, and another study reported that dietary intake was improved in the intervention group [32]. One study reported no significant intervention effects on stress or dietary knowledge scores [38]. However, another study reported that emotional functioning significantly increased after the intervention [37].

\section{Discussion}

\section{Principal Findings}

This study identified the effects of ICT intervention on obesity-related outcomes in children and adolescents in RCTs through a systematic literature review and meta-analysis. We found that although ICT intervention is not significantly effective in reducing $\mathrm{BMI}, \mathrm{BW}, \mathrm{BMI} \mathrm{z}$-score, $\mathrm{WC}$, and \% $\mathrm{BF}$, it does work in certain groups.

A subgroup analysis by intervention type revealed that the intervention effect was statistically significant only in the web or web plus intervention for BMI, BW, and WC. This result is consistent with those of previous studies that showed that web-based health programs are effective in managing obesity. Meta-analyses have demonstrated that web-based interventions are effective in achieving weight loss in adults [41,42]. Several systematic reviews have explored the use of web-based interventions for the prevention or treatment of obesity and related conditions in pediatric populations [20,22]. In addition, a meta-analysis demonstrated that mobile-based interventions are effective in achieving weight loss $[43,44]$ and reducing BMI [45-47] in adults. However, there has been no meta-analysis of mobile-based obesity intervention studies in children. Several systematic reviews have indicated that mobile-based interventions in obesity treatment programs have a modest effect on weight control [47-51]. However, these effects are inconsistent. Owing to the nature of mobile use, access is possible from anywhere; hence, the possibility of giving formal responses in situations where participants are unprepared to take certain actions cannot be ruled out. Therefore, additional RCTs and meta-analyses targeting the weight-loss effect of mobile-based interventions in children and adolescents are warranted.

Another subgroup analysis by comparator type revealed that the intervention effect was significant only in the lifestyle modification comparator rather than in the control comparator for BMI and WC. A previous meta-analysis [52] that analyzed the weight loss effect of circuit training demonstrated that focusing on circuit training alone rather than adding other lifestyle interventions to circuit training is effective. Among the RCTs included in our meta-analysis, 2 [35,38] had the lifestyle modification comparator. In these 2 studies, the comparator group did not focus on one lifestyle, intervened in various lifestyle habits, and the number of contacts was not frequent. Recognizing that they are undergoing an intervention, participants tend to act passively, hoping to elicit changes to their lifestyle habits; therefore, where there is no intensive intervention, the intervention can be counterproductive. However, as there may be other factors influencing the research results, additional studies comparing ICT with a comparator group focusing on one lifestyle are needed.

For WC, a significant intervention effect was also obtained when the comparator type was control. Among the RCTs included in our meta-analysis, 2 [32,40] had the control comparator. However, the difference in sample size was large between the 2 studies; thus, the results of the subgroup were not different from those of one study [40], which had a large sample size. Therefore, it is unreasonable to interpret this as a subgroup result.

The intervention effect was significant only in the intervention involving both boys and girls for BMI and WC. Among the RCTs included in our meta-analysis, one evaluated boys only [40], another evaluated girls only [39], and the other 8 did not differentiate between the sexes of the participants. Sex differences in response to ICT interventions are likely to be because of differences in participation. In cases where boys and girls participate together, the resultant mutual competition can increase participation, which subsequently increases the effect of the intervention. Several studies have compared the sex-dependent effects of school-based physical activity interventions [53-56]. However, to date, no meta-analysis has shown sex differences in the effects of ICT intervention on children with obesity. Therefore, additional RCTs are needed to investigate the sex-dependent weight loss effects of ICT interventions.

In addition, the intervention effect was significant only in the intervention involving obesity only for BMI and WC. Participants with normal weight or overweight status may be less motivated than with participants with obesity, which may dilute the overall effect. It is encouraging to identify meaningful results from studies solely involving children and adolescents with obesity. In other words, significant results can be obtained if ICT intervention is implemented in children and adolescents with obesity. Further well-designed ICT intervention studies targeting children and adolescents with obesity should be conducted.

When both parents and children were involved in children less than 10 years of age, a reduction in BMI $z$-score was observed. In the same situation, other outcome variables did not show a statistically significant effect; however, considering the BMI $z$-score only, ICT could be used as a means of preventing obesity-related outcomes by intervening in early childhood with the involvement of parents. Therefore, there is a need for additional long-term RCTs involving high-quality ICT interventions targeting parents and children under 10 years of age.

Previous studies have demonstrated that mHealth programs or internet technology have a higher attrition rate than conventional face-to-face methods $[57,58]$. However, the average attendance rate of the participants in this meta-analysis was $86.5 \%$. Children with obesity are often ashamed because of social stigma and may face prejudice against weight [59]. Moreover, because children and teenagers have a high level of interest in and concentration with electronic devices, access through mobile means or the internet can be more efficient [60]. Therefore, the effect of obesity intervention through mHealth programs or 
internet technology is considered superior because of enhanced accessibility and an increased participation rate compared with conventional interventions such as the face-to-face method.

The advantage of childhood-obesity management using mHealth programs or internet technology is that it is possible to operate programs led by peer participants and provide real-time feedback. In particular, by presenting a mission aimed at improving dietary habits or increasing exercise among peers as well as providing rankings or rewards, it is possible to induce mutual participation through goodwill competition. However, among the studies included in this study, no study was conducted in a manner that induced competition among participants. Therefore, further research on the participation rates is imperative.

\section{Strengths}

Our meta-analysis had the following strengths: First, to the best of our knowledge, this is the first meta-analysis to assess the association between ICT intervention and weight loss in children and adolescents. Second, we examined the differences in weight-loss effects among subgroups according to the type of intervention, type of comparator, target participants, mean age, sex, BMI status, and follow-up period. We found that ICT intervention is effective for weight loss in the web intervention, lifestyle modification comparator, intervention involving both boys and girls, and intervention involving obesity only. Finally, the heterogeneity among the included RCTs was low.

\section{Limitations}

Our meta-analysis has several limitations. Although the meta-analysis found a moderate effect size that was statistically significant, as not many studies were included in the meta-analysis, generalization of the study results is limited because of potential publication bias. However, there were no small study effects according to the Egger regression test results. In addition, many of the studies were of short duration, making it unclear whether weight loss was sustained in the long term. Although the clinically significant threshold for weight loss was not always achieved across the studies, studies of longer duration might have found clinically significant weight loss. Therefore, further evidence is necessary to confirm this hypothesis. Outcome measurements based on participants' self-reports for many studies were recorded using mobile apps and websites and the lack of comments on the reliability of the measurement method using mobile apps can limit the results analysis. In addition, no RCT has focused on sex differences in the use of mHealth programs and internet technology. Therefore, future research should investigate the sex-dependent weight loss effects of ICT interventions.

\section{Conclusions}

The meta-analysis results for children and adolescents with obesity who participated in the web intervention program confirmed significant effects on BMI reduction compared with the lifestyle modification intervention. Evidence from the meta-analysis identified internet technology as a useful tool for weight loss in children and adolescents with obesity.

\section{Authors' Contributions}

YGS and JP wrote the manuscript; YGS, JP, and MJP analyzed the data and interpreted the results; YGS participated in the design of the study; all authors reviewed the manuscript, contributed to the discussion, and read and approved the final manuscript.

\section{Conflicts of Interest}

None declared.

\section{Multimedia Appendix 1}

Risk of bias assessment and forest plots.

[DOCX File, 223 KB-Multimedia Appendix 1]

\section{References}

1. Fact sheets: obesity and overweight. World Health Organization. 2021. URL: https://www.who.int/news-room/fact-sheets/ detail/obesity-and-overweight [accessed 2021-03-01]

2. Ogden CL, Carroll MD, Lawman HG, Fryar CD, Kruszon-Moran D, Kit BK, et al. Trends in obesity prevalence among children and adolescents in the United States, 1988-1994 through 2013-2014. J Am Med Assoc 2016 Jun 07;315(21):2292-2299. [doi: 10.1001/jama.2016.6361] [Medline: 27272581]

3. Tirosh A, Shai I, Afek A, Dubnov-Raz G, Ayalon N, Gordon B, et al. Adolescent BMI trajectory and risk of diabetes versus coronary disease. N Engl J Med 2011 Apr 07;364(14):1315-1325. [doi: 10.1056/nejmoa1006992]

4. Skinner AC, Perrin EM, Moss LA, Skelton JA. Cardiometabolic risks and severity of obesity in children and young adults. N Engl J Med 2015 Oct;373(14):1307-1317. [doi: 10.1056/NEJMoa1502821] [Medline: 26422721]

5. Twig G, Yaniv G, Levine H, Leiba A, Goldberger N, Derazne E, et al. Body-Mass Index in 2.3 million adolescents and cardiovascular death in adulthood. N Engl J Med 2016 Jun 23;374(25):2430-2440. [doi: 10.1056/nejmoa1503840]

6. Mühlig Y, Antel J, Föcker M, Hebebrand J. Are bidirectional associations of obesity and depression already apparent in childhood and adolescence as based on high-quality studies? A systematic review. Obes Rev 2016 Mar 18;17(3):235-249. [doi: 10.1111/obr.12357] [Medline: 26681065] 
7. Stice E, Shaw H, Marti CN. A meta-analytic review of obesity prevention programs for children and adolescents: the skinny on interventions that work. Psychol Bull 2006 Sep;132(5):667-691 [FREE Full text] [doi: 10.1037/0033-2909.132.5.667] [Medline: 16910747]

8. Haynos AF, O'Donohue WT. Universal childhood and adolescent obesity prevention programs: review and critical analysis. Clin Psychol Rev 2012 Jul;32(5):383-399. [doi: 10.1016/j.cpr.2011.09.006] [Medline: 22681912]

9. Seo DC, Sa J. A meta-analysis of obesity interventions among U.S. minority children. J Adolesc Health 2010 Apr;46(4):309-323. [doi: 10.1016/j.jadohealth.2009.11.202] [Medline: 20307819]

10. Luttikhuis HO, Baur L, Jansen H, Shrewsbury VA, O'Malley C, Stolk RP, et al. Interventions for treating obesity in children. Cochrane Database Syst Rev 2009(1):CD001872. [doi: 10.1002/14651858.CD001872.pub2] [Medline: 19160202]

11. Kelly AS, Fox CK, Rudser KD, Gross AC, Ryder JR. Pediatric obesity pharmacotherapy: current state of the field, review of the literature and clinical trial considerations. Int J Obes (Lond) 2016 Jul 26;40(7):1043-1050 [FREE Full text] [doi: 10.1038/ijo.2016.69] [Medline: 27113643]

12. Inge TH, Courcoulas AP, Jenkins TM, Michalsky MP, Helmrath MA, Brandt ML, Teen-LABS Consortium. Weight loss and health status 3 years after bariatric surgery in adolescents. N Engl J Med 2016 Jan 14;374(2):113-123 [FREE Full text] [doi: 10.1056/NEJMoa1506699] [Medline: 26544725]

13. Lenhart A. Teens, social media and technology overview 2015. Pew Research Center. 2015. URL: https://www. pewresearch.org/internet/2015/04/09/teens-social-media-technology-2015/ [accessed 2021-03-01]

14. Mascheroni G, Olafsson K, Cuman A, Dinh T, Haddon L, Jørgensen H, et al. Mobile internet access and use among European children: initial findings of the Net Children Go Mobile project. In: Net Children Go Mobile Initial Findings Report. Milan, Italy: Educatt; 2013.

15. Roberts DF, Foehr UG, Rideout V. Generation M: Media in the lives of 8-18 year-olds. Kaiser Family Foundation. 2005. URL: https://www.kff.org/wp-content/uploads/2013/01/generation-m-media-in-the-lives-of-8-18-year-olds-report.pdf [accessed 2021-03-01]

16. Brenner V. Psychology of computer use: XLVII. Parameters of Internet use, abuse and addiction: the first 90 days of the Internet Usage Survey. Psychol Rep 1997 Jun 01;80(3 Pt 1):879-882. [doi: 10.2466/pr0.1997.80.3.879] [Medline: 9198388$]$

17. Fang K, Mu M, Liu K, He Y. Screen time and childhood overweight/obesity: a systematic review and meta-analysis. Child Care Health Dev 2019 Sep;45(5):744-753. [doi: 10.1111/cch.12701] [Medline: 31270831]

18. World Health Organization. mHealth: New Horizons for Health through Mobile Technologies: Based on the Findings of the Second Global Survey on eHealth (Global Observatory for eHealth Series, Volume 3). Geneva: World Health Organization; 2020:1-111.

19. Silva BM, Rodrigues JJ, de la Torre Díez I, López-Coronado M, Saleem K. Mobile-health: a review of current state in 2015. J Biomed Inform 2015 Aug;56:265-272. [doi: 10.1016/j.jbi.2015.06.003] [Medline: 26071682]

20. Nguyen B, Kornman KP, Baur LA. A review of electronic interventions for prevention and treatment of overweight and obesity in young people. Obes Rev 2011 May;12(5):298-314. [doi: 10.1111/j.1467-789X.2010.00830.x] [Medline: 21348921]

21. Antwi F, Fazylova N, Garcon MC, Lopez L, Rubiano R, Slyer JT. The effectiveness of web-based programs on the reduction of childhood obesity in school-aged children: a systematic review. JBI Libr Syst Rev 2012;10(42 Suppl):1-14. [doi: 10.11124/jbisrir-2012-248] [Medline: 27820152]

22. An JY, Hayman LL, Park YS, Dusaj TK, Ayres CG. Web-based weight management programs for children and adolescents: a systematic review of randomized controlled trial studies. ANS Adv Nurs Sci 2009;32(3):222-240. [doi: 10.1097/ANS.0b013e3181b0d6ef] [Medline: 19707091]

23. Casanova G, Zaccaria D, Rolandi E, Guaita A. The effect of information and communication technology and social networking site use on older people's well-being in relation to loneliness: review of experimental studies. J Med Internet Res 2021 Mar 01;23(3):e23588 [FREE Full text] [doi: 10.2196/23588] [Medline: 33439127]

24. Higgins JPT, Thomas J, Chandler J, Cumpston M, Li T, Page MJ, et al. Cochrane Handbook for Systematic Reviews of Interventions. 2nd Edition. Chichester (UK): John Wiley \& Sons; 2019.

25. Akers J. Systematic Reviews: CRD's Guidance for Undertaking Reviews in Health Care. York: CRD, University of York; 2009.

26. Moher D, Liberati A, Tetzlaff J, Altman DG, PRISMA Group. Preferred reporting items for systematic reviews and meta-analyses: the PRISMA statement. Br Med J 2009 Jul 21;339:b2535 [FREE Full text] [doi: 10.1136/bmj.b2535] [Medline: 19622551$]$

27. Higgins JP, Thompson SG, Deeks JJ, Altman DG. Measuring inconsistency in meta-analyses. Br Med J 2003 Sep 6;327(7414):557-560 [FREE Full text] [doi: 10.1136/bmj.327.7414.557] [Medline: 12958120]

28. DerSimonian R, Laird N. Meta-analysis in clinical trials. Control Clin Trials 1986 Sep;7(3):177-188. [doi: 10.1016/0197-2456(86)90046-2] [Medline: 3802833]

29. Egger M, Smith G, Schneider M, Minder C. Bias in meta-analysis detected by a simple, graphical test. Br Med J 1997 Sep 13;315(7109):629-634 [FREE Full text] [doi: 10.1136/bmj.315.7109.629] [Medline: 9310563]

30. Prochaska JO, Velicer WF. The transtheoretical model of health behavior change. Am J Health Promot 1997;12(1):38-48. [doi: 10.4278/0890-1171-12.1.38] [Medline: 10170434] 
31. Johansson L, Hagman E, Danielsson P. A novel interactive mobile health support system for pediatric obesity treatment: a randomized controlled feasibility trial. BMC Pediatr 2020 Sep 23;20(1):447 [FREE Full text] [doi: 10.1186/s12887-020-02338-9] [Medline: $\underline{32967638]}$

32. Chai LK, Collins CE, May C, Ashman A, Holder C, Brown LJ, et al. Feasibility and efficacy of a web-based family telehealth nutrition intervention to improve child weight status and dietary intake: a pilot randomised controlled trial. J Telemed Telecare 2019 Jul 31:1357633X19865855. [doi: 10.1177/1357633X19865855] [Medline: 31364474]

33. Nyström C, Sandin S, Henriksson P, Henriksson H, Maddison R, Löf M. A 12-month follow-up of a mobile-based (mHealth) obesity prevention intervention in pre-school children: the MINISTOP randomized controlled trial. BMC Public Health 2018 May 24;18(1):658 [FREE Full text] [doi: 10.1186/s12889-018-5569-4] [Medline: 29793467]

34. Bruñó A, Escobar P, Cebolla A, Álvarez-Pitti J, Guixeres J, Lurbe E, et al. Home-exercise childhood obesity intervention: a randomized clinical trial comparing print versus web-based (Move It) platforms. J Pediatr Nurs 2018 Sep;42:79-84. [doi: 10.1016/j.pedn.2018.04.008] [Medline: 29747957]

35. Rerksuppaphol L, Rerksuppaphol S. Internet based obesity prevention program for thai school children - a randomized control trial. J Clin Diagn Res 2017;11(3):7-11. [doi: 10.7860/jcdr/2017/21423.9368]

36. Mameli C, Brunetti D, Colombo V, Bedogni G, Schneider L, Penagini F, et al. Combined use of a wristband and a smartphone to reduce body weight in obese children: randomized controlled trial. Pediatr Obes 2018 Feb;13(2):81-87. [doi:

10.1111/ijpo.12201] [Medline: 27900849]

37. Nawi AM, Jamaludin FI. Effect of internet-based intervention on obesity among adolescents in Kuala Lumpur: a school-based cluster randomised trial. Malays J Med Sci 2015;22(4):47-56 [FREE Full text] [Medline: 26715908]

38. Abraham AA, Chow WC, So HK, Yip BH, Li AM, Kumta SM, et al. Lifestyle intervention using an internet-based curriculum with cell phone reminders for obese Chinese teens: a randomized controlled study. PLoS One 2015;10(5):e0125673 [FREE Full text] [doi: 10.1371/journal.pone.0125673] [Medline: 25946465]

39. Nollen NL, Mayo MS, Carlson SE, Rapoff MA, Goggin KJ, Ellerbeck EF. Mobile technology for obesity prevention: a randomized pilot study in racial- and ethnic-minority girls. Am J Prev Med 2014 Apr;46(4):404-408 [FREE Full text] [doi: 10.1016/j.amepre.2013.12.011] [Medline: 24650843]

40. Smith JJ, Morgan PJ, Plotnikoff RC, Dally KA, Salmon J, Okely AD, et al. Smart-phone obesity prevention trial for adolescent boys in low-income communities: the ATLAS RCT. Pediatrics 2014 Sep;134(3):723-731 [FREE Full text] [doi: 10.1542/peds.2014-1012] [Medline: 25157000]

41. Sherrington A, Newham JJ, Bell R, Adamson A, McColl E, Araujo-Soares V. Systematic review and meta-analysis of internet-delivered interventions providing personalized feedback for weight loss in overweight and obese adults. Obes Rev 2016 Jun;17(6):541-551 [FREE Full text] [doi: 10.1111/obr.12396] [Medline: 26948257]

42. Beleigoli AM, Andrade AQ, Cançado AG, Paulo MN, Diniz MF, Ribeiro AL. Web-based digital health interventions for weight loss and lifestyle habit changes in overweight and obese adults: systematic review and meta-analysis. J Med Internet Res 2019 Jan 08;21(1):e298 [FREE Full text] [doi: 10.2196/jmir.9609] [Medline: 30622090]

43. Khokhar B, Jones J, Ronksley PE, Armstrong MJ, Caird J, Rabi D. Effectiveness of mobile electronic devices in weight loss among overweight and obese populations: a systematic review and meta-analysis. BMC Obes 2014;1:22 [FREE Full text] [doi: 10.1186/s40608-014-0022-4] [Medline: 26217509]

44. Kim HN, Seo K. Smartphone-based health program for improving physical activity and tackling obesity for young adults: a systematic review and meta-analysis. Int J Environ Res Public Health 2019 Dec 18;17(1):15 [FREE Full text] [doi: 10.3390/ijerph17010015] [Medline: 31861359]

45. Flores Mateo G, Granado-Font E, Ferré-Grau C, Montaña-Carreras X. Mobile phone apps to promote weight loss and increase physical activity: a systematic review and meta-analysis. J Med Internet Res 2015;17(11):e253 [FREE Full text] [doi: 10.2196/jmir.4836] [Medline: 26554314]

46. Park SH, Hwang J, Choi YK. Effect of mobile health on obese adults: a systematic review and meta-analysis. Healthc Inform Res 2019 Jan;25(1):12-26 [FREE Full text] [doi: 10.4258/hir.2019.25.1.12] [Medline: 30788177]

47. Liu F, Kong X, Cao J, Chen S, Li C, Huang J, et al. Mobile phone intervention and weight loss among overweight and obese adults: a meta-analysis of randomized controlled trials. Am J Epidemiol 2015 Mar 1;181(5):337-348. [doi: 10.1093/aje/kwu260] [Medline: 25673817]

48. Wang Y, Xue H, Huang Y, Huang L, Zhang D. A systematic review of application and effectiveness of mHealth interventions for obesity and diabetes treatment and self-management. Adv Nutr 2017 May;8(3):449-462. [doi: 10.3945/an.116.014100] [Medline: 28507010]

49. Bacigalupo R, Cudd P, Littlewood C, Bissell P, Hawley MS, Woods HB. Interventions employing mobile technology for overweight and obesity: an early systematic review of randomized controlled trials. Obes Rev 2013 Apr;14(4):279-291 [FREE Full text] [doi: 10.1111/obr.12006] [Medline: 23167478]

50. Dounavi K, Tsoumani O. Mobile health applications in weight management: a systematic literature review. Am J Prev Med 2019 Jun;56(6):894-903 [FREE Full text] [doi: 10.1016/j.amepre.2018.12.005] [Medline: 31003801]

51. Stephens J, Allen J. Mobile phone interventions to increase physical activity and reduce weight: a systematic review. J Cardiovasc Nurs 2013;28(4):320-329 [FREE Full text] [doi: 10.1097/JCN.0b013e318250a3e7] [Medline: 22635061] 
52. Seo YG, Noh HM, Kim SY. Weight loss effects of circuit training interventions: a systematic review and meta-analysis. Obes Rev 2019 Nov 19;20(11):1642-1650. [doi: 10.1111/obr.12911] [Medline: 31322317]

53. Vizcaíno V, Aguilar F, Gutiérrez R, Martínez M, López M, Martínez S, et al. Assessment of an after-school physical activity program to prevent obesity among 9- to 10-year-old children: a cluster randomized trial. Int J Obes (Lond) 2008 Jan 25;32(1):12-22. [doi: 10.1038/sj.ijo.0803738] [Medline: 17895883]

54. Bugge A, El-Naaman B, Dencker M, Froberg K, Holme IM, McMurray RG, et al. Effects of a three-year intervention: the Copenhagen School Child Intervention Study. Med Sci Sports Exerc 2012 Jul;44(7):1310-1317. [doi: 10.1249/MSS.0b013e31824bd579] [Medline: 22297806]

55. Aguilar F, Martínez-Vizcaíno V, López M, Martínez M, Gutiérrez R, Martínez S, et al. Impact of an after-school physical activity program on obesity in children. J Pediatr 2010 Jul;157(1):36-42. [doi: 10.1016/j.jpeds.2009.12.046] [Medline: $\underline{20227726}]$

56. Martínez-Vizcaíno V, Sánchez-López M, Notario-Pacheco B, Salcedo-Aguilar F, Solera-Martínez M, Franquelo-Morales $\mathrm{P}$, et al. Gender differences on effectiveness of a school-based physical activity intervention for reducing cardiometabolic risk: a cluster randomized trial. Int J Behav Nutr Phys Act 2014 Dec 10;11(1):154 [FREE Full text] [doi: 10.1186/s12966-014-0154-4] [Medline: 25491026]

57. Druce KL, Dixon WG, McBeth J. Maximizing engagement in mobile health studies: lessons learned and future directions. Rheum Dis Clin North Am 2019 May;45(2):159-172 [FREE Full text] [doi: 10.1016/j.rdc.2019.01.004] [Medline: 30952390]

58. Okorodudu DE, Bosworth HB, Corsino L. Innovative interventions to promote behavioral change in overweight or obese individuals: a review of the literature. Ann Med 2015 May;47(3):179-185 [FREE Full text] [doi: 10.3109/07853890.2014.931102] [Medline: 25011006]

59. Sjöberg RL, Nilsson KW, Leppert J. Obesity, shame, and depression in school-aged children: a population-based study. Pediatrics 2005 Sep 01;116(3):389-392. [doi: 10.1542/peds.2005-0170] [Medline: 16140683]

60. Tate EB, Spruijt-Metz D, O'Reilly G, Jordan-Marsh M, Gotsis M, Pentz MA, et al. mHealth approaches to child obesity prevention: successes, unique challenges, and next directions. Transl Behav Med 2013 Dec;3(4):406-415 [FREE Full text] [doi: 10.1007/s13142-013-0222-3] [Medline: 24294329]

\author{
Abbreviations \\ \%BF: percentage body fat \\ BW: body weight \\ ICT: information and communication technology \\ mHealth: mobile health \\ PRISMA: Preferred Reporting Items for Systematic Reviews and Meta-Analyses \\ RCT: randomized controlled trial \\ WC: waist circumference \\ WMD: weighted mean difference
}

Edited by R Kukafka, G Eysenbach; submitted 22.03.21; peer-reviewed by J Piqueras, C Carrion; comments to author 26.04.21;
revised version received 02.05.21; accepted 03.10.21; published 17.11.21
Please cite as:
Park J, Park MJ, Seo YG
Effectiveness of Information and Communication Technology on Obesity in Childhood and Adolescence: Systematic Review and
Meta-analysis
J Med Internet Res $2021 ; 23(11): e 29003$
URL: $\underline{\text { https://Www.jmir.org/2021/11/e29003 }}$
doi: $\underline{10.2196 / 29003}$
PMID: $\underline{34787572}$

(CJihyun Park, Mi-Jeong Park, Young-Gyun Seo. Originally published in the Journal of Medical Internet Research (https://www.jmir.org), 17.11.2021. This is an open-access article distributed under the terms of the Creative Commons Attribution License (https://creativecommons.org/licenses/by/4.0/), which permits unrestricted use, distribution, and reproduction in any medium, provided the original work, first published in the Journal of Medical Internet Research, is properly cited. The complete bibliographic information, a link to the original publication on https://www.jmir.org/, as well as this copyright and license information must be included. 